\title{
Sialic Acid Storage Disease
}

National Cancer Institute

\section{Source}

National Cancer Institute. Sialic Acid Storage Disease. NCI Thesaurus. Code C85067.

A rare, autosomal recessive lysosomal storage disease caused by mutations in the SLC17A5 gene. It primarily affects the nervous system. Signs and symptoms include developmental delay, intellectual disability, hypotonia, failure to thrive, seizures, and ataxia. 